\title{
A influência do estímulo e do vínculo familiar no desenvolvimento de crianças com síndrome de Down
}

\author{
The influence of stimulation and family bonding on the development of children with \\ Down syndrome
}
La influencia de la estimulación y la vinculación familiar en el desarrollo de los niños con síndrome de Down

Caroline Porto Aguiar ${ }^{1 *}$, Caio Oslo Oliveira Cardoso², Dayanna da Silva Carvalho Faria², Joyce Oliveira Matos $^{3}$, Lucas Adriano de Oliveira Silva ${ }^{2}$, Layla Sales Silva Rabêlo², Murillo Cursino de Castro Silva ${ }^{4}$, Shaina Medeiros de Figueredo², Lorena Leão Gomes Leahy², Michela Macedo Lima Costa1.

\section{RESUMO}

Objetivo: Analisar como o estímulo e o vínculo familiar influenciam no desenvolvimento de crianças com Síndrome de Down. Revisão bibliográfica: O desenvolvimento de indivíduos portadores da síndrome sofre influência de acordo com a qualidade do cuidado, educação e experiência que the são oferecidos. Assim, esses serão motivados pelos recursos que estão disponíveis e pelas atitudes das pessoas com as quais convivem. Através da brincadeira a criança desenvolve suas capacidades motoras, verbal e cognitivas. Ou seja, ela é uma forma de assimilação funcional e repetitiva que desenvolve hábitos e esquemas sensóriomotores. Além disso, torna-se necessário a orientação da família, para que esses estejam mais envolvidos e seguros no processo do desenvolvimento da criança, e possam planejar métodos criativos, lúdico para que em casa iniciem a estimulação precoce. Quando esta intervenção precoce se focaliza no binômio pais-criança, os efeitos são mais favoráveis, garantindo a continuidade do tratamento em casa. Considerações finais: Com isso, o estímulo precoce e adequado além de um ambiente familiar favorável pode contribuir para esse desenvolvimento.

Palavras-chave: Síndrome de Down, Vínculo familiar, Estímulo.

\begin{abstract}
Objective: To analyze how stimulation and family bonding influence the development of children with Down syndrome. Bibliographic review: The development of individuals with the syndrome is influenced according to the quality of care, education and experience offered to them. Thus, these will be motivated by the resources that are available and the attitudes of the people they live with. Through play, children develop their motor, verbal and cognitive abilities. In other words, it is a form of functional and repetitive assimilation that develops sensory-motor habits and schemes. In addition, it is necessary to guide the family, so that they are more involved and secure in the child's development process, and can plan creative, playful methods so that they can start early stimulation at home. When this early intervention focuses on the parent-child binomial, the effects are more favorable, ensuring continuity of treatment at home. Final considerations: With this, early and adequate stimulation, in addition to a favorable family environment, can contribute to this development.
\end{abstract}

Keywords: Down syndrome, Family attachment, Stimulus.

\footnotetext{
1 Faculdade Santo Agostinho (FASA), Vitória da Conquista - BA. *E-mail: carolinepop1@ @otmail.com

${ }^{2}$ Faculdade Santo Agostinho de Itabuna (FASAI), Itabuna - BA.

3 Universidade José do Rosário Vellano (UNIFENAS-BH), Belo Horizonte - MG.

${ }^{4}$ Centro Universitário FG (UniFG), Guanambi - BA.
} 


\section{RESUMEN}

Objetivo: Analizar cómo la estimulación y el vínculo familiar influyen en el desarrollo de los niños con síndrome de Down. Revisión bibliográfica: El desarrollo de las personas con el síndrome se ve influido de acuerdo con la calidad de la atención, la educación y la experiencia que se les ofrece. Así, estos estarán motivados por los recursos disponibles y las actitudes de las personas con las que conviven. A través del juego, los niños desarrollan sus habilidades motoras, verbales y cognitivas. Es decir, es una forma de asimilación funcional y repetitiva que desarrolla hábitos y esquemas sensorio-motores. Además, es necesario orientar a la familia, para que esté más involucrada y segura en el proceso de desarrollo del niño, y pueda planificar métodos creativos y lúdicos para que pueda iniciar la estimulación temprana en casa. Cuando esta intervención temprana se centra en el binomio padre-hijo, los efectos son más favorables, lo que garantiza la continuidad del tratamiento en el hogar. Consideraciones finales: Con esto, una estimulación temprana y adecuada, además de un entorno familiar favorable, puede contribuir a este desarrollo.

Palabras clave: Síndrome de Down, Apego familiar, Estímulo.

\section{INTRODUÇÃO}

A Síndrome de Down (SD) é uma das modificações genéticas envolvendo cromossomos mais conhecidas, trata-se de uma desordem genética e a causa é a trissomia do cromossomo do cromossomo 21 (HENDGES VM, et al., 2021). Esta síndrome tem incidência em torno de um a cada 700 nascidos vivos e ocorre com maior frequência em mulheres que se tornam mães com idade superior a trinta e cinco anos. Além disso, no gênero masculino esse evento ocorre mais tardiamente, quando a paternidade acontece após os cinquenta e cinco anos de idade (SAITO YC, et al., 2021)

A condição genética do cromossomo 21 extra, acarreta características físicas atípicas no indivíduo, além de um atraso no desenvolvimento. Porém, apesar de tais fatores, quando é se feito um atendimento de qualidade e um estímulo adequado, crianças com SD têm potencial para um desenvolvimento semelhante a crianças típicas, bem como uma melhor inclusão social (BRASIL, 2013).

Ademais, devido principalmente os avanços na área da saúde, em destaque na cirurgia cardíaca, a expectativa de vida das pessoas com SD aumentou consideravelmente a partir do século XX. Esse aumento da sobrevida e a descoberta da capacidade desse grupo motivou a criação de diversos programas voltados para a educação, futuro profissional e melhoria na qualidade de vida (HANNUM JSS, et al., 2018).

Cada vez mais a sociedade está preparada para inclusão das pessoas com SD. Porém, apesar do conhecimento adquirido nos últimos anos, ainda não é possível determinar o nível de desenvolvimento e autonomia que crianças com SD terão. Entretanto, a maioria dos profissionais que atuam no cuidado desse grupo, apoiam uma maior dedicação nas áreas da educação, estimulação precoce e inclusão social, principalmente através de familiares, que possuem um papel imprescindível nesse desenvolvimento (BRASIL, 2013).

O desenvolvimento de indivíduos portadores da síndrome sofre influência de acordo com a qualidade do cuidado, educação e experiência que lhe são oferecidos. Ou seja, esses serão motivados pelos recursos que estão disponíveis e pelas atitudes das pessoas com as quais convivem (FERREIRA M, et al., 2019).

As crianças portadoras da SD possuem características comuns, como atraso no desenvolvimento cognitivo e motor, sendo capazes de executar os mesmos movimentos que crianças típicas, porém mais tardiamente, além da capacidade de desenvolver estágios avançados de raciocínio e desenvolvimento. No entanto, para esta evolução é necessária uma estimulação coerente com a condição, um ambiente e apoio familiar adequados e um bom acompanhamento multidisciplinar (SOUZA JM e VERÍSSIMO MLÓR, 2015).

Dessa forma, se faz necessário entender como a estimulação e o ambiente familiar agem para um melhor desenvolvimento de crianças com SD. Sendo a família a base fundamental desse processo, pois ela é capaz de promover as primeiras experiências emocionais e de aprendizagem da criança, contribuindo para esse avanço. Com isso, o estímulo precoce e adequado, além de um ambiente favorável podem contribuir para esse desenvolvimento (ARAKI IPM e BAGAGI PS, 2014). 
Assim, esse constructo teve como objetivo analisar como o estímulo e o vínculo familiar influenciam no desenvolvimento de crianças com Síndrome de Down.

\section{REVISÃO BIBLIOGRÁFICA}

\section{Desenvolvimento de crianças típicas}

O Desenvolvimento Infantil (DI) é uma etapa fundamental para um adequado desenvolvimento humano, observando-se a moldagem da arquitetura cerebral, logo nos primeiros anos, a partir da interação entre herança genética e influências do meio em que a criança vive. O desenvolvimento de forma distinta entre desenvolvimento físico, cognitivo e psicossocial é uma forma de facilitar o estudo do desenvolvimento humano. Porém, apesar dessa divisão, ressalta que esses aspectos estão interligados e influenciam em conjunto durante a vida do indivíduo (RABELO PRS, et al., 2020).

O desenvolvimento fisiológico humano depende da sua interação com o meio social no qual está inserido. Logo, o desenvolvimento infantil terá como mediadores as pessoas nas quais esse grupo possui uma maior relação, destacando sua família e educadores que delimitam e atribuem significados a sua realidade. (OLIVEIRA CM, 2021).

Para o sucesso de um desenvolvimento é essencial a integridade dos diversos órgão e sistemas que contribuem para o processo, em especial o sistema nervoso, por participar de toda organização funcional que o indivíduo irá vivenciar. Há um crescimento e amadurecimento do tecido nervoso nos primeiros anos de vida, logo, esse período é bastante vulnerável aos agravos ambientais que podem acarretar prejuízo neste processo. No entanto, nessa época, o grau de plasticidade cerebral é maior, portanto, crianças respondem melhor a estímulos que recebem e as intervenções, quando necessárias (MOURA ER, 2018).

Em relação a aquisição motora, os recém-nascidos possuem um padrão muito imaturo, apresentado um reflexo tônico cervical assimétrico, predominando tônus flexor nos membros. Os movimentos desenvolvidos nessa faixa etária são, geralmente, reflexos que possuem controle por partes primitivas do cérebro. Durante os primeiros meses, há uma diminuição do tônus flexor e este é substituído pelo extensor. Essa evolução ocorre no sentido craniocaudal, sendo os membros inferiores os últimos a desenvolverem. Porém, a partir dos 6 meses, a criança não irá possuir mais predomínio por um padrão de tônus, alternando entre eles. Dessa forma, ela já consegue desempenhar atividades como rolar, e posteriormente, após dissociação dos movimentos da cintura escapular e da pelve, consegue sentar (REGIS MS, et al., 2018).

O desenvolvimento motor possui algumas regras para se seguir, entre elas, que ele ocorra no sentido craniocaudal, proximodistal, e a partir de atividades mais simples, para as mais complexas. Dessa forma, a primeira musculatura a se desenvolver é a ocular, onde possibilita a fixação do olhar. Posteriormente, a cervical, para aquisição da sustentação do tronco e na sequência, as extremidades superiores adquirem um apoio da musculatura, que corrobora para o processo de engatinhar da criança, e por volta dos 9 meses, está assume posição ortostática, seguindo uma evolução para a deambulação (TRINDADE AS e NASCIMENTO MA, 2016).

Em relação ao desenvolvimento motor fino, se dá no sentido proximodistal. Ao nascer, as crianças mantem as mãos fechas grande parte do tempo, e no decorrer do desenvolvimento, por volta dos 3 meses, ficam abertas por um período maior e conseguem agarrar objetos, isso devido à redução do tônus flexor. Por volta do $5^{\circ}$ ao $6^{\circ}$ mês, conseguem segurar um objeto por vontade própria e iniciam o movimento de pinça, que sofre evolução até chegar à aquisição da polpa com polpa (RAMOS BB e MÜLLER AB, 2019).

No que se refere à interação social, o olhar juntamente com o sorriso, representam meios de comunicação e estão presentes desde o nascer, porém, até a $6^{a}$ semana de vida é possível observar o "sorriso social" motivado por estímulos, em destaque pela face materna. Esta ainda relata que por volta dos 60 meses 0 sorriso não costuma ser mais "social" e a criança não reage assim a qualquer adulto, passando a distinguir um familiar de um estranho. Nesse sentindo, ela começa a expressar diversos comportamentos, como medo e recusa (PEREIRA LV e OLIVEIRA EMP, 2015). 
Em relação a linguagem, nos primeiros anos de vida, o bebê se expressa através da mimica facial e, principalmente através do choro. Por volta do $2^{\circ}$ e $3^{\circ}$ mês, a criança começa a emissão de arrulhos, e aos $6^{\circ}$ meses sons silabais. Por volta de 9 e 10 meses, começa realmente as primeiras palavras com entonação semelhantes à linguagem do meio em que vive. A linguagem gestual surge por volta do $2^{\circ}$ semestre de vida e vai ser consequência da interação com os adultos e o meio. Nesse período a criança já está apta para apontar e obedecer a alguns comandos verbais, como por exemplo, bater palmas. No decorrer do desenvolvimento, por volta dos 12 meses surgem as primeiras palavras, e aos 18 meses ela inicia frases simples, seguindo para um aumento do repertório de palavras (REGIS MS, et al., 2018).

\section{Fisiopatologia da Síndrome de Down}

A SD foi descrita pela primeira vez em 1866 pelo médico britânico John Landgon Dawn, entretanto, apenas 100 anos depois que foi descoberto o cromossomo 21 a mais nas pessoas portadoras de SD, essa descoberta foi feita pelo geneticista Jérôme Lejeune em 1958 (SANTOS LS e MOTA BGN, 2021).

As características das pessoas com SD, como face achatada, olhos bem espaçados e testa ampla, eram semelhantes às de povos nativos da Mongólia, logo os portadores da síndrome foram muitas vezes denominados como mongoloides pela população da época. Além disso, há um número maior de casos observados em mulheres com idade avançada, comprovando a relação de quanto maior a idade, maior a probabilidades de erros genéticos, podendo resultar em alterações como a SD (PIETRICOSKI LB e JUSTINA LAD, 2020).

O material genético de um ser humano possui 46 cromossomos, sendo 22 pares autossômicos e 1 par sexual, em que nas mulheres possui representação de 46XX e nos homens 46XY. Quando uma alteração, seja ela numérica ou estrutural, ocorre no conjunto dos cromossomos, pode levar a danos e até impedir o desenvolvimento embrionário. Em relação a SD ocorre um cromossomo 21 a mais no conjunto diploide. Isso ocasiona uma anormalidade cromossômica que acaba levando uma expressão genética irregular no desenvolvimento embrionário, levando a alterações no organismo do indivíduo (CORRÊA RA e STROPARO E, 2015).

A trissomia do cromossomo 21 ocorre devido uma anormalidade na gametogênese, onde pode acontecer uma distribuição desigual dos cromossomos e consequentemente acarretar um material genético desbalanceado com a presença de um cromossomo extra. A união desse material desbalanceado com um gameta normal levará a um embrião com uma trissomia parcial do cromossomo 21 (CORRÊA RA e STROPARO E, 2015). A trissomia do cromossomo 21 pode ocorrer de três formas, a parcial, o mosaicismo genético e por translocação robertsoniana (JUNIOR JBS, et al., 2020).

O mosaicismo genético é caracterizado quando a trissomia ocorre em parte das células do indivíduo, conservando outra parte, devido a não-disjunção na mitose de algumas células do embrião. Essa forma acontece mais raramente, correspondendo a apenas $2 \%$ dos casos. Por último tem-se a terceira forma de trissomia, a translocação robertsoniana, neste fenômeno o cromossomo extra é herdado devido a fusão com outro cromossomo (GONÇALVES TM e KARASAWA MMG, 2021; JUNIOR JBS, et al., 2020)

Em relação ao grau de desenvolvimento dos indivíduos com SD, esses diferentes tipos de trissomia do cromossomo 21 não acarretam diferenças, exceto no mosaicismo, onde não afeta todas as células, logo se observa um menor comprometimento desse desenvolvimento. Portanto, as diferenças encontradas em indivíduos portadores da Síndrome estão mais relacionadas com determinantes sócias, do que os genéticos (GONÇALVES TM e KARASAWA MMG, 2021). No início da gestação, por volta da 11ª semana, os sinais e sintomas dessa anormalidade já podem ser notados, a partir do aumento da transluscência nucal e um menor tamanho do osso nasal identificados na ultrassonografia (COSTA AG, et al., 2020).

\section{Características dos indivíduos com SD}

Existe uma enorme variedade de características das pessoas portadoras da SD, as principais e mais encontradas são: Deficiência intelectual, apresentando um grau de retardo mental. Além disso, pessoas com SD também podem apresentar, problemas cardíacos; dificuldades na fala; pregas epicânticas; língua grande 
e sulcada; orelhas pequenas e com dobras nas pontas, com implantação mais baixa; pescoços mais curtos com dobras na região posterior e apenas uma prega palmar nas mãos. A pele pode ser mais sensível e os cabelos na grande maioria são lisos e finos (BRASIL, 2013).

Outros problemas que podem ser apresentados na SD, são os distúrbios tireoidianos, o envelhecimento precoce, predisposição as leucemias e a alteração na coluna cervical, devido à instabilidade da articulação atlanto-axial (CORRÊA RA e STROPARO E, 2015). Além disto, indivíduos portadores da SD possuem restrições intrínsecas como a hipotonia muscular e hipermobilidade articular e tais fatores contribuem para 0 atraso do desenvolvimento motor (RAMOS BB e MÜLLER AB, 2019).

De modo geral, é possível observar padrões atípicos para o controle postural, locomoção e até mesmo para manipulação de objetos. $\mathrm{O}$ andar é comum nos portadores de SD, porém realizam com a base alargada e com maior oscilação do tronco e cabeça e apresentam um maior grau de flexão nos quadris, nos joelhos e no tronco (SILVA AC, 2014).

Em relação a cognição, crianças com SD possuem dificuldades no processo de transmissão de alguns circuitos neuronais, acarretando problemas de atenção, no processamento auditivo-vocal e na memória a curto e a médio prazo. Além disso, apresentam dificuldades nos processos de cálculos, pensamentos abstratos e correlação. E destacam os déficits sensoriais, principalmente o auditivo, como fator de influência para comunicação (COELHO C, 2016).

\section{Estímulo e o vínculo familiar no desenvolvimento de crianças com SD}

Muitos familiares sofrem um impacto ao receberem a notícia que o bebê nasceu com SD, inicialmente muitas mães não aceitam, podendo ter dificuldades como rejeitar o filho. A partir do momento que é dado o diagnóstico, até a família chegar ao ponto da aceitação ela passa por um longo processo com diversos sentimentos de choque, negação, raiva, revolta e rejeição, até que a convivência começa a despertar sentimentos de afeto, carinho, amor (HANNUM JSS, et al., 2018).

Assim, torna-se necessário a orientação da família, para que esses estejam mais envolvidos e seguros no processo do desenvolvimento da criança, e possam planejar métodos criativos, lúdico para que em casa iniciem a estimulação precoce (PEREIRA LV e OLIVEIRA EMP, 2015). As atividades da vida cotidiana na família possibilitarão à criança, seja ela com desenvolvimento típico ou portadora da SD, oportunidades para aprender e se desenvolver através do modelo, da participação de atividades em conjunto com os familiares, da realização com acompanhamento de responsáveis e de diversas outras formas capazes de possibilitarem a aprendizagem. Além disso, a relação familiar produz importantes efeitos no desenvolvimento cognitivo, socioemocional e na linguagem da criança (SEHN AS, et al., 2019).

É notório que a privação de estímulos prazerosos logo no início da vida, nas crianças, pode levar a dificuldades no processamento de informações sensoriais, assim como um retardo no seu desenvolvimento motor. Logo, costuma-se intervir precocemente quando um bebê apresenta desordens psicossomáticas ou de desenvolvimento. Quando iniciada até o $4^{\circ}$ mês de idade do bebê antes do estabelecimento atípico de modelos posturais e movimentos, é considerada intervenção precoce. Logo, é recomendada como forma de ter maior interação da criança com o ambiente, alcançando reações motoras próximas ao padrão da normalidade e evitando o desenvolvimento incomum de movimento e postura (GONÇALVES TM e KARASAWA MMG, 2018).

Quando esta intervenção precoce se focaliza no binômio pais-criança, os efeitos são mais favoráveis, garantindo a continuidade do tratamento em casa. Ou seja, essa é mais efetiva se a família for um elemento ativo na prática do tratamento. Ademais, a estimulação que incluí envolvimento dos pais, indicam um resultado mais positivo no desenvolvimento da criança com SD, comparando com a intervenção que não envolveram pais (SEHN AS, et al., 2019). Além disso, torna-se essencial, pois o trabalho desenvolvido em uma área reflete sofre as demais. Além das especialidades médicas, inclui-se na equipe áreas da Fonoaudiologia, Psicologia e Fisioterapia (GOIS IKF e JUNIOR FFUS, 2018).

Em relação ao desenvolvimento motor, estes profissionais intervêm com o intuito de estabelecer a funcionalidade do movimento, exercitando a crianças no sentido de torná-la hábil a posturas e movimentos 
viáveis, através do incentivo de experiências motoras adequadas. Com isso, para proporcionar um aprendizado motor com bons resultados o trabalho de reparação deve ser intenso e constante, e se necessário e possível realizado diariamente (MARINHO MFS, 2018).

Além disso, é importante individualizar os programas voltados para a estimulação, pois esses programas têm como objetivo atender individualmente cada criança. Assim, tem que ser levado em consideração as diferenças de cada criança, como, por exemplo, os fatores genéticos e as vivencias de cada criança, pois eles podem interferir na prática do programa de estimulação. Além disso, é importante que essas atividades ocorram semanalmente para melhor desenvolver as crianças (GOIS IKF e JUNIOR FFUS, 2018).

Os estímulos envolvendo as funções sensoriais auxiliarão na compreensão das crianças sobre o que ocorre ao seu redor. O lugar mais estimulante para esse desenvolvimento é o chão, sendo a posição prona a melhor empregada, pois possibilita um fortalecimento dos músculos da coluna e auxilia o levantamento da cabeça. Além disso, todos os sentidos devem ser solicitados: pelo tato, experimentam diferentes consistências, a visão possibilita acompanhar os movimentos de objetos e pessoas; a audição distingue diferentes sons (MATTOS BM e BELLANI CDF, 2010).

A estimulação da criança para virar a cabeça lateralmente em busca de um som possibilita um melhor desenvolvimento do seu sistema auditivo, e com a introdução de diferentes sons, exercitará sua atenção. A princípio será notado os ruídos mais fortes, porém, no decorrer do seu desenvolvimento será possível distinguir os sons familiares, como as vozes dos pais. Logo em seguida, poderá perceber sons de distâncias maiores (PELOSIA MB, et al., 2020).

A fala é uma atividade que deve ser estimulada, no entanto é necessário orientar aos pais e responsáveis da criança com SD que a velocidade de processamento difere entre as crianças e isto não significa que a estimulação esteja sendo inadequada. Sendo assim, é de suma importância trabalhar a fala com o acompanhamento profissional do fonoaudiólogo para estimula-la (PEREIRA LV e OLIVEIRA EMP, 2015). A elaboração de um ambiente recreativo também é de suma importância para o desenvolvimento geral da criança. $O$ ato de brincar auxilia no processo de desenvolvimento, socialização e aprendizagem (DAMASCENO BCE, et al., 2017).

Através da brincadeira a criança desenvolve suas capacidades motoras, verbal e cognitivas. Ou seja, ela é uma forma de assimilação funcional e repetitiva que desenvolve hábitos e esquemas sensório-motores. Ainda que a brincadeira do portador de SD seja semelhante à da criança típica, ela tende a ser menos explorativa, sendo importante estimulá-lo com brincadeiras, por exemplo, jogos com regras, para que se tenha uma participação efetiva através do brincar, no trabalho de estimulação de sua sensibilidade, postura e equilíbrio (PELOSIA MB, et al., 2020).

\section{CONSIDERAÇÕES FINAIS}

Dessa forma, se faz necessário entender como a estimulação e o ambiente familiar agem para um melhor desenvolvimento de crianças com SD. Sendo a família a base fundamental desse processo, pois ela é capaz de promover as primeiras experiências emocionais e de aprendizagem da criança, contribuindo para esse avanço. Com isso, o estímulo precoce e adequado além de um ambiente favorável pode contribuir para esse desenvolvimento. Desse modo, pesquisas como esta visam possibilitar uma reflexão sobre a influência precoce de pessoas com SD e o vínculo familiar no desenvolvimento dessas crianças.

\section{REFERÊNCIAS}

1. ANHÃO PPG, et al. Interação social de crianças com Síndrome de Down na educação infantil. Revista Brasileira de Educação Especializada, 2018; 16(1): 31-46.

2. ARAKI IPM, BAGAGI PS. Síndrome de Down e seu desenvolvimento motor. Revista Científica Eletrônica de Pedagogia, 2014; (23): 1-6.

3. COELHO C. A síndrome de Down. Revista Luso-Brasileira de Psicologia, 2016; 1-14.

4. CORREA RA, STROPARO E. Síndrome de Down: Uma revisão. Revista Eletrônica Biociências, Biotecnologia e Saúde, 2015; (13): 40-51. 
5. COSTA AG, et al. Critérios para a realização da ultrassonografia no primeiro trimestre da gestação baseados nos guidelines isuog. Revista Brasileira de Ultrassonografia, 2020; 28(29): 49-56.

6. DAMASCENO BCE, et al. A importância do brincar para o desenvolvimento da criança com Síndrome Down. Society and Development, 2017; 4(2): 142-152.

7. FERREIRA M, et al. Repercussões do Diagnóstico de Síndrome de Down na Perspectiva Paterna. Psicologia: Ciência e Profissão, 2019; 39: 1-14.

8. GOIS IKF, JUNIOR FFUS. Estimulação precoce em crianças com síndrome de Down. Revista Fisioterapia Brasil, 2018; 19(5): 684-692.

9. GONÇALVES TM, KARASAWA MMG. "Muta-ação: a proposta de um jogo lúdico sobre mutações e síndromes genéticas nas disciplinas de biologia molecular e genética clássica. Revista Arquivos do Mudi, 2021; 25(1): 44-65.

10. HANNUM JSS, et al. Impacto do Diagnóstico nas Famílias de Pessoas com Síndrome de Down: Revisão da Literatura. Revista Pensando Famílias, 2018; 22(2): 121-136.

11. HENDGES VM, et al. Avaliação do desenvolvimento psicomotor de crianças com Síndrome de Down. Revista Neurociências, 2021; 29: 1-26.

12. JÚNIOR JBS, et al. Especificação e Desenvolvimento de um ChatBot para Apoio ao Atendimento a Pessoas com Trissomia 21. Revista Ibérica de Sistemas e Tecnologias de Informação, 2020; (42): 238-244.

13. MARINHO MFS. A intervenção fisioterapêutica no tratamento motor da síndrome de down: uma revisão bibliográfica. Revista Campo do Saber, 2018; 4(1): 67-74.

14. MATTOS BM, BELLANI CDF. A importância da estimulação precoce em bebês portadores de síndrome de Down: revisão de literatura. Revista Brasileira de Terapias e Saúde, 2010; 1(1): 51-63.

15. MOURA ER. Síndrome de Down e aprendizagem em diálogo com as neurociências: limites e possibilidades na escola regular. Revista Vozes dos Vales, 2018; (14): 1-21.

16. OLIVEIRA CM. Aspectos relevantes da psicomotricidade na educação infantil. Revista autênticos, $2021 ; 1(1)$ : 67-82.

17. PELOSIA MB, et al. Atividades terapêuticas ocupacionais desenvolvidas com crianças e pré-adolescentes com síndrome de Down. Cadernos Brasileiros de Terapia Ocupacional, 2020; 28(2): 511-524.

18. PEREIRA LV, OLIVEIRA EMP. Influência do entorno familiar no desempenho comunicativo de crianças com Síndrome de Down. Revista Speech, Language, Hearing Sciences and Education Journal (CEFAC), 2015; 17(1): 177-183.

19. PIETRICOSKI LB, JUSTINA LAD. História da construção do conhecimento sobre a Síndrome de Down no século XIX e início do século XX. Research, Society and Development, 2020; 9(6): e165963574.

20. RABELO PRS, et al. Avaliação do desenvolvimento físico de lactentes no contexto da atenção primária à saúde. Revista Enfermagem Atual In Derme, 2020; 92(30): 267-277.

21. RAMOS BB, MÜLLER AB. Marcos motores e sociais de crianças com síndrome de down na estimulação precoce. Revista Interdisciplinar Ciências Médicas, 2019; 4(1): 37-43.

22. REGIS MS, et al. Estimulação fonoaudiológica da linguagem em crianças com síndrome de Down. Revista Centro de Especialização em Fonoaudiologia Clínica, 2018; 20(3): 271-280.

23. SAITO YC, et al. Hábitos alimentares de indivíduos com síndrome de Down. Advances in Nutritional Sciences, 2021; (1): e0332021-9.

24. SANTOS LS, MOTA BGN. Os desafios do professor para as práticas inclusivas de alunos com síndrome de down nos anos iniciais do ensino fundamental. Revista Educação \& Ensino, 2021; 5(1): 125-147.

25. SEHN AS, et al. Interação mãe/pai-criança com síndrome de Down: revisão sistemática da literatura. Revista Contextos Clínicos, 2019; 12(1): 153-185.

26. SILVA, AC. A utilização da equoterapia no tratamento da síndrome de down: uma revisão sistemática. Revista Gestão, Tecnologia e Ciências, 2014; 3(6): 68-77.

27. SOUZA JM, VERÍSSIMO MLÓR. Desenvolvimento infantil: análise de um novo conceito. Revista latino-americana de enfermagem, 2015; 23(6): 1097-1104.

28. TRINDADE AS, NASCIMENTO MA. Avaliação do Desenvolvimento Motor em Crianças com Síndrome de Down. Revista Brasileira de Educação Especializada, 2016; 22(4): 577-88. 